\title{
Correlation of the distribution of antioxidant enzyme concentrations in blood serum and heart tissue in rats
}

\author{
Pavel Boriskin ${ }^{1}$, Olga Gulenko ${ }^{2}$, Anatoly Devyatkin ${ }^{1}$, Rufia Karimova ${ }^{1}$, Victor Leonov ${ }^{2}$, and Olga Pavlova ${ }^{3, *}$ \\ ${ }^{1}$ Kazan State Academy of Veterinary Medicine named after N.E. Bauman, Kazan 420029, Russia \\ ${ }^{2}$ Reaviz Medical University, Samara 443001, Russia \\ ${ }^{3}$ TestGen LLC, Ulyanovsk 432072, Russia
}

\begin{abstract}
Cardiovascular diseases have been the leading cause of death worldwide for many years. In recent years, new cardiovascular disease markers have been sought that can improve the diagnosis and treatment of this broad and prognostically unfavorable group of diseases. The efforts of many researchers are aimed at detecting changes in the level of enzymes in the lipid peroxidation system, which are antioxidants, as possible mechanisms underlying the development of cardiovascular disease. The imbalance between the intensification of free-radical oxidation caused by active oxygen forms and the activity of the body's protective antioxidant system leads to serious disturbances: disorganization of cellular structures, changes in their functional activity. The article presents the study of the interrelation of the concentration distribution of enzymes of the lipid peroxidation system - antioxidants in blood serum and heart tissues of white non-pedigree rats. The correlation coefficients of Spearman, the gamma of correlation and Kendel Tau revealed a reliable presence of weak correlation between the concentration of glutathione peroxidase in blood serum and heart tissues (Spearman $\mathrm{R}=0.18$ at $\mathrm{p} \leq 0.029408$, Gamma $=0.14$ at $\mathrm{p} \leq 0.018701$; Kendall Tau $=0.13$ at $\mathrm{p} \leq 0.018701)$.
\end{abstract}

\section{Introduction}

The highest prevalence of cardiovascular diseases is the cause of increased mortality and disability in the working population in economically developed countries. The search for the root causes of etymology of various diseases leads to the concept of oxidative stress, which develops against the background of disturbance of homeostatic balance of the body under the influence of endo- and exogenous factors.

Intensification of oxidation-reduction processes enhances the formation of active oxygen species such as peroxynitrite, superoxide anion and others, which accumulating in large quantities disturb the process of synthesis of collagen and proteoglycans, connective tissue, cause microcirculatory disorders and provoke vascular endothelial dysfunction, depriving it of the ability to adapt to changes in hemodynamics, increasing vasoconstriction, which in turn leads to the development of a number of diseases: atherosclerosis, ischemic heart disease, arterial hypertension, diabetes mellitus [1].

One of the frequent variants of cardiovascular disease is the formation of chronic heart failure, and the more risk factors for this pathology have been identified, the more intense the oxidation processes in the body [2]. Free radicals cause damage to cardiomyocytes, disrupt the structural modification of the lipid layer with subsequent remodeling of myocardium, thereby impairing its contractile functions, providing a direct negative inotropic effect [3-8].

Enzymes (superoxide dismutase (SOD), glutathione peroxidase (GP), glutathione reductase (GR), catalase) playing an antioxidant role in neutralizing high-activity superoxide anions play an important role in establishing homeostatic balance, hydrogen peroxides and fatty acid hydroperoxides in the initial stage of lipid peroxidation (LOA) processes and preventing accumulation of toxic molecular products.

One of the main components of the body's antioxidant protection is a group of metal enzymes, superoxide dismutase (SOD), which catalyzes the dismutation reaction of superoxide radical anions and maintains the concentration of these radicals in the cell at a low level $[9,10]$.

In addition, SOD in general reduces the probability of the formation of singlet oxygen, the activity of which is 3-4 orders of magnitude higher than that of superoxide radical anions [11]. For the first time superoxide dismutase was described in 1969 by the scientists McCord and Fridovich. Depending on the type of metal ion, this enzyme is subdivided into several isoforms: copper and zinc-containing superoxide dismutase (Cu,Zn-SOD), Mn-SOD (found in mammalian mitochondria matrix) and $\mathrm{Fe}-\mathrm{SOD}$ (found in prokaryotes). Copper and zinc-containing superoxide dismutase present in aerobic cell cytosol and liver

Corresponding author: casiopeya13@mail.ru 
lysosomes of warm-blooded animals is more widespread and most active.

In the enzyme molecule, zinc and copper ions, interacting with each other, are in such proximity that the resulting changes in the environment of one ion affect the environment of another. Copper ions catalyze the activity of the enzyme, but there is no direct correlation between copper absorption and ODS activity. Zinc ions play a structural role by transforming the protein into an active center of the enzyme.

Single-valued anions (chlorine, hydroxyl) are competitive enzyme inhibitors that bind copper ions in the active site. The enzyme is inhibited by cyanide. Hydrogen peroxide inactivation of SOD is accompanied by luminescence and reduction of cytochrome $\mathrm{C}$. However, hydrogen peroxide acts as an enzyme reducing agent at low concentrations. The reconstituted enzyme is quite resistant to oxygen.

There is also an extracellular form of superoxide dismutase, which was first isolated in 1982. It is a glycoprotein consisting of two dimers connected by a disulfide bridge. Each of the 4 subunits has a molecular weight of about $30 \mathrm{kDa}$ and contains copper and zinc ions. All enzyme subunits have the same amino acid sequence. However, the extracellular and cytoplasmic $\mathrm{Cu}-\mathrm{Zn}-\mathrm{SOD}$ subunits are distinguished by their amino acid composition, antigenic properties, gene locus, amino acid encoding sequence of the apoenzyme and catalytic activity.

If the cytoplasmic $\mathrm{Cu}-\mathrm{Zn}-\mathrm{SOD}$ is a homodimer, each subunit of which has four residues of cysteine, the extracellular superoxide dismutase is a tetramer, the subunits of which contain six residues of cysteine [12].

Extracellular ODS is present mainly in extracellular spaces. It was found in fibroblasts and glial cells [13] and is the main isoenzyme of plasma, lymph, synovial fluid [14]. High level of extracellular superoxide dismutase is found in lungs, heart, kidneys and placenta. It is inhibited by cyanide, inactivated by hydrogen peroxide, and iron ions block enzyme secretion [15].

The mechanism of SOD functioning includes sequential reduction and oxidation of metal ions of variable valence in the active center of the enzyme. ODS is an endogenous acceptor of free oxygen radicals, the excessive accumulation of which in the cell is important in the development of a number of oxygen dependent pathological processes (hypoxia, inflammation, intoxication, etc.) ODS removes superoxide radicals and prevents the formation of other more dangerous to the body free radicals: hydroxyl radical and singlet oxygen. In addition, SOD prevents the accumulation of neutrophils in the inflammation zone, which secrete significant amounts of lysosomal enzymes that destroy nearby tissues [16-18].

Extracellular SOD is the main regulator of nitrogen monoxide (NO) bioactivity, which reacts with oxygen anion-radicals to form highly toxic peroxynitrite. Nitrogen monoxide is synthesized by endothelial cells, macrophages and neutrophils, is an endothelial relaxing factor, maintains the required level of vasodilation and has antiplatelet activity, preventing thrombosis.
The content and activity of isoenzymes in organs and tissues vary. In humans, the total amount of $\mathrm{Cu}-, \mathrm{Zn}$ SOD can reach $3900 \mathrm{mg}$. High levels of $\mathrm{Cu}-$, $\mathrm{Zn}-\mathrm{ODS}$ and Mn-ODS are found in the liver, in red blood cells, which allows using blood as a source of enzyme excretion.

The content of these isoforms in organs and tissues varies less than that of extracellular $\mathrm{Cu}-, \mathrm{Zn}-\mathrm{ODS}$, which is inferior to them in quantity [19].

With age, there is a significant decrease in the specific activity of cytoplasmic superoxide dismutase. A decrease in the catalytic activity of the enzyme is observed.

Decrease of superoxidase activity in the postmitochondrial fraction of the brain in contrast to mitochondria during aging is associated with weakening of the enzyme biosynthesis and with typical age-related posttranslational changes caused by oxidative modification of the polypeptide protein chain. According to Sahoo D, the activation of lipid peroxidation, the increase in the amount of hydrogen peroxide, and the decrease in the level of glutathione accompanied by the decrease in the activity of ODS, glutathione peroxidase, and glutathione reductase in the testicular mitochondria of rats with age. $\mathrm{K}$. and co-authors may indicate a weakening of the antioxidant system and influence such physiological functions as steroidogenesis and spermatogenesis [20]. Also, the activity of the enzyme may decrease with deficiency of metal ions in the body. Oxidative stress can both reduce and increase the activity of ODS in comparison with the physiological norm.

According to the research data, the reduction of superoxidase activity contributes to the strengthening of hypertension, development of arthritis, diabetes, diabetic retinopathy, diabetic nephropathy [21, 22] and other pathological processes accompanied by the development of oxidative stress.

All of the above suggests that the assessment of superoxide-sumtase activity in blood and tissues is necessary to determine the causes and mechanisms of development of a pathological process in the body and to propose methods of treatment of diseases.

Glutathione reductase/glutathione peroxidase (GR/GP) system is the most important component of antioxidant protection of the organism [23], which maintains the intensity of free radical processes at the stationary level [24]. Due to the functioning of the GR/GP system in mammalian cells, the detoxification of hydroperoxides and peroxides, which are the main source of hydroxyl radicals, is ensured [25]. Glutathione reductase is considered to be an enzymatic link in the endogenous antioxidant protection system. The substrate for glutathione reductase is oxidized glutathione, which it converts into reduced glutathione. Enzyme activity increases with increased concentration of reduced forms of peridinucleotides and oxidized glutathione. Thus, glutathione reductase - glutathione peroxidase forms a closed antiperoxidase complex in which peroxidase neutralizes peroxides to hydrogen and water, while glutathione is oxidized and glutathione reductase restores oxidized glutathione, turning it into a substrate for glutathione peroxidase activity. 
Glutathione peroxidase (GP) capable of catalyzing the peroxide detoxification reaction without formation of free radicals using reduced glutathione - $\gamma$-glutamyl cysteinyl glycine (GSH) as a hydrogen donor, as well as synthesizing and reducing low molecular weight thiols [26].

Warm-blooded animals are characterized by the presence of several forms of glutathione peroxidases, depending on the organ or tissue detected. Most often, this enzyme is a homotetrameter that includes selenocysteine, which is necessary for an enzymatic reaction.

According to some research data, plants, bacteria and protozoa insects do not have seleno-dependent HP, but it functions in all kinds of fish, worms and mammals. This is an evolutionary young enzyme, the acquisition of which has become an important advantage for vertebrates and may have led to the transformation of selenium from an ordinary element into a bioelement. Selenium deficiency in the body causes an increase in the active oxygen forms in cells, and, consequently, leads to a weakening of the immune system, the development of diseases associated with numerous changes in the structure and functions of biopolymers (proteins, nucleic acids and lipids).

Dynamics of GP formation allows drawing conclusions about the mechanism of compensation of disadaptation shifts, about features of metabolism at regulation of processes of free radical oxidation and to predict development and dynamics of pathological conditions in warm-blooded organisms.

One of the indicators of the efficiency of the warmblooded organism is the activity of catalase. Catalase is a chromoprotein, a tetramer of four polypethyde chains including more than 500 amino acids, and also includes four groups of porphyry heme capable of oxidizing active oxygen forms. Oxidized gem is a prosthetic group of catalase. It is the first link of intracellular protection against active oxygen species [27].

The main function of the catalase is the neutralization of anion-radical O2-, hydroxyl radical, unsaturated fatty acid radicals (lipopericisi), the splitting of hydrogen peroxide formed in the process of cellular oxygen, breathing into molecular oxygen and water. At the same time, catalase does not require energy for activation and is a highly active enzyme.

Thus, catalase is one of the main elements of antioxidant protection of the body, providing a dynamic balance between the formation of active oxygen species and their elimination. Catalase in large quantities is formed in organelles (peroxisomes) and cytoplasm of cells. Homothermal organisms have a high content of this representative of oxidoreductase class in the liver, kidneys and red blood cells.

In the development of pathologies the leading processes are membrane-destructive processes, including the processes of free radical oxidation, which determine the degree of endotoxemia.

By the number and dynamics of catalase activity one can indirectly judge the state of the organism, the degree of oxidative stress and the level of endogenous intoxication, which are the result of pathological internal or external processes. For example, an indicator of some cancers and neuropathologies (Parkinson's disease) is a decrease in catalase activity in the kidneys and liver $[28,29]$. The activity of antioxidant protection enzymes, including catalase, is associated with the adaptation of heart tissue to hypoxia, which reduces the probability of heart attack and ischemia.

Decrease in catalase activity occurs when there is an excess of methionine, cystine, copper, zinc [30].

Despite the rather deep study of cardiovascular system pathologies, data on the functional activity of myocardial cells under destructive effects and oxidative stress are still insufficient.

Thus, the aim of our study is to study the relationship between the distribution of POL-AO enzyme concentrations in blood serum and heart tissues of white non-pedigree rats.

In order to achieve this goal, the following tasks had to be solved: to determine the concentration of catalase, superoxide dismutase (SOD), glutathione peroxidase (GP) and glutathione reductase (GR) in blood serum and heart tissues of white non-pedigreed rats; to reveal the relationship between the distribution of antioxidant enzymes in blood serum and heart tissues of rats.

\section{Materials and methods}

The study was carried out on white, non-patented, healthy male rats of the same month of birth, weighing $180-200 \mathrm{~g}$, in the amount of 150 pieces, which were kept in the vivarium under standard conditions.

The study was carried out in accordance with the rules of laboratory practice in the Russian Federation: the order of the Ministry of Health of the USSR № 755 from 12.08.1977, the order of the Ministry of Health of the Russian Federation № 267 from 19.06.2003, the law "On the protection of animals from cruelty", Chapter V, Art. 104679-GD from 01.12.1999.

The activity of catalase was determined by the standard method of Korolyuk M.A. [31]. The activity of superoxide dismutase was determined by the method of V.S. Gurevich and co-authors [32]. The activity of glutathione peroxidase was determined by the method of V.M. Moyn. Glutathione reductase activity was determined spectrophotometrically at a wavelength of $340 \mathrm{~nm}$ [33].

Enzyme concentrations were studied in heart tissue and blood serum. For this purpose, rats were ethically murdered under etheric anesthesia by decapitation, and then the necessary tissues were extracted, which (except for blood serum) were washed with physiological solution and immediately frozen. Homogenates were prepared by mechanical shredding of tissues weighing 1 $\mathrm{g}$ with $9 \mathrm{ml}$ of tris buffer ( $\mathrm{pH} \mathrm{7.4)}$, at a rate of $5000 \mathrm{rpm}$ in a vessel with double walls, constantly cooled by running water [33].

Digital material was statistically processed by nonparametric Spearman correlation analysis, as well as by using gamma correlation coefficients and Kendella Tau. 


\section{Results}

As a result of experiments an array of numerical concentrations of catalase, SOD, GP, and GR blood serum and heart tissues of white non-pedigree rats was obtained. The obtained results were statistically processed (Table 1). At the first stage of the statistical analysis the check for compliance with the normal distribution of antioxidant enzyme concentrations in blood serum and heart tissues of rats was carried out. For this purpose Kolmogorov-Smirnov single-sample criterion was used. As a result, it was found that the distribution of antioxidant enzymes in blood serum and rat heart tissues did not correspond to normal. In connection with that at the further statistical processing we applied nonparametric methods of analysis.

In order to estimate the correlation between the distribution of antioxidant enzyme concentrations in blood serum and heart tissues of small experimental animals, we studied the correlations within the observation group using the nonparametric Spearman correlation coefficient (Table 2), as well as using the gamma correlation coefficients and Kendell Tau (Table 3).

According to the data presented in Table 2, a weak correlation between glutathione peroxidase concentration in blood serum and heart tissues $(0.18$ at $p \leq 0.029408)$ can be traced. Since no correlation between the distribution of antioxidant enzyme concentrations in blood serum and rat heart tissues was found using the Spearman correlation coefficient, it was decided to carry out an analysis using the gamma correlation criteria and Kendella Tau (Table 3).

According to the data presented in Table 3 it is seen that the direct reliable correlation between the concentration of glutathione peroxidase in blood serum and heart tissues of rats was revealed when studying the distribution of concentrations of antioxidant enzymes in blood serum and heart tissues of white non-pedigreed rats: Gamma 0.14 at $\mathrm{p} \leq 0.018701$; Kendall Tau 0.13 at $\mathrm{p} \leq 0.018701$.

Table 1. Distribution of antioxidant enzyme concentrations in blood serum and heart tissue of white rats.

\begin{tabular}{|c|c|c|c|c|c|c|c|c|c|}
\hline $\begin{array}{l}\text { Descriptive statistics } \\
\text { of merged groups }\end{array}$ & $\mathrm{N}$ & M & $\mathrm{Me}$ & Min & Max & 25 Perc & 75 Perc & 10 Perc & 90 Perc \\
\hline \multicolumn{10}{|c|}{ Catalase } \\
\hline Blood serum & 150 & 19.72 & 19.60 & 17.40 & 22.10 & 18.90 & 20.40 & 18.35 & 21.40 \\
\hline Heart & 150 & 29.25 & 29.40 & 27.10 & 30.90 & 28.50 & 30.10 & 28.10 & 30.60 \\
\hline \multicolumn{10}{|c|}{ Superoxide dismutase } \\
\hline Blood serum & 150 & 29.01 & 29.10 & 27.30 & 30.70 & 28.40 & 29.60 & 28.00 & 30.20 \\
\hline Heart & 150 & 96.96 & 96.80 & 94.80 & 98.90 & 96.30 & 97.70 & 95.60 & 98.45 \\
\hline \multicolumn{10}{|c|}{ Glutathione peroxidase } \\
\hline Blood serum & 150 & 123.50 & 123.50 & 122.10 & 125.10 & 123.10 & 124.20 & 122.60 & 124.70 \\
\hline Heart & 150 & 272.04 & 271.90 & 270.70 & 273.80 & 271.50 & 272.60 & 271.25 & 273.10 \\
\hline \multicolumn{10}{|c|}{ Glutathione reductase } \\
\hline Blood serum & 150 & 70.75 & 70.80 & 69.40 & 71.80 & 70.40 & 71.20 & 69.80 & 71.60 \\
\hline Heart & 150 & 53.44 & 53.40 & 52.10 & 54.90 & 53.10 & 53.80 & 52.60 & 54.30 \\
\hline
\end{tabular}

Table 2. Spearman Correlation Factor for the distribution of antioxidant enzyme concentrations in blood serum and rat heart tissue and $\mathrm{p}$.

\begin{tabular}{|l|l|c|c|c|}
\hline \multicolumn{1}{|c|}{ Enzyme } & \multicolumn{1}{c|}{$\begin{array}{c}\text { Spearman correlation } \\
\text { in all combined dimensions }\end{array}$} & Valid N & Spearman R & p-level \\
\hline Catalase & Blood serum \& heart & 150 & -0.072130 & 0.380397 \\
\hline Superoxide dismutase & Blood serum \& heart & 150 & -0.079408 & 0.334076 \\
\hline Glutathioperoxidase & Blood serum \& heart & $\mathbf{1 5 0}$ & $\mathbf{0 . 1 7 7 8 9 8}$ & $\mathbf{0 . 0 2 9 4 0 8}$ \\
\hline Glutathione reductase & Blood serum \& heart & 150 & 0.059254 & 0.471360 \\
\hline
\end{tabular}

Table 3. Gamma and Kendella Tau correlation coefficients for the distribution of antioxidant enzymes in blood serum and rat heart tissue

\begin{tabular}{|c|c|c|c|c|c|}
\hline \multicolumn{6}{|c|}{ Correlation Gamma Ratio Coefficient } \\
\hline \multicolumn{6}{|c|}{ MD pairwise deleted Marked correlations are significant at $\mathrm{p}<0.05000$} \\
\hline Enzyme & $\begin{array}{c}\text { Correlation in all } \\
\text { combined measurements }\end{array}$ & Valid N & Gamma & $\mathbf{Z}$ & p-level \\
\hline Catalase & Blood serum \& heart & 150 & -0.050561 & -0.88672 & 0.375229 \\
\hline Superoxide dismutase & Blood serum \& heart & 150 & -0.056349 & -0.985468 & 0.324394 \\
\hline Glutathioperoxidase & Blood serum \& heart & 150 & 0.136009 & 2.351434 & 0.018701 \\
\hline Glutathione reductase & Blood serum \& heart & 150 & 0.045872 & 0.780006 & 0.435388 \\
\hline \multicolumn{6}{|c|}{ Kendall Tau's correlation coefficient } \\
\hline \multicolumn{6}{|c|}{ MD pairwise deleted Marked correlations are significant at $p<, 05000$} \\
\hline Enzyme & $\begin{array}{c}\text { Correlation in all } \\
\text { combined measurements }\end{array}$ & Valid N & Kendall Tau & $\mathrm{Z}$ & p-level \\
\hline Catalase & Blood serum \& heart & 150 & -0.048831 & -0.88672 & 0.375229 \\
\hline Superoxide dismutase & Blood serum \& heart & 150 & -0.054268 & -0.985468 & 0.324394 \\
\hline Glutathioperoxidase & Blood serum \& heart & 150 & 0.129490 & 2.351434 & 0.018701 \\
\hline Glutathione reductase & Blood serum \& heart & 150 & 0.042954 & 0.780006 & 0.435388 \\
\hline
\end{tabular}




\section{Conclusion}

Thus, all three methods of nonparametric correlation analysis used to assess the relationship between the distributions of antioxidant enzymes within the physiological norm revealed a correlation between the concentration of glutathione peroxidase in blood serum and heart tissues of white rats.

\section{References}

1. S. Ferderbar, E.C. Pereira, E. Apolinário, et al., Cholesterol oxides as biomarkers of oxidative stress in type 1 and type 2 diabetes mellitus, Diabetes Metab. Res. Rev., 23(1), 35-42 (2007)

2. K.K. Griendling, G.A. FitzGerald, Oxidative stress and cardiovascular injury, Part I: basic mechanisms and in vivo monitoring of ROS, Circulat., 108, 1912-1916 (2003)

3. A.N. Zakirova, R.R. Gabidullin, N.E. Zakirova, Clinicogemodynamic effects of carvedylol, influence on lipid peroxidation and inflammation markers in patients with CHD, Heart failure, 7, 1 (2006)

4. T. Heitzer, T.K. Schlinzig, et al., Endothelial dysfunction, oxidative stress and risk of cardiovascular events in patients with coronary disease, Circulat., 104, 263-268 (2001)

5. U. Landmesser, S. Spiekermann, S. Dikalov et al., Vascular oxidative stress and endothelial dysfunction in patients with chronic heart failure: role of xanthine-oxidase and extracellular superoxide dismutase, Circulat., 106, 3073-3078 (2002)

6. F.J. Giordano, Oxygen, oxidative stress, hypoxia, and heart failure, J. Clin. Invest., 115, 500-508 (2005)

7. A. Lopez Farre, S. Casado, Heart failure, redox alterations, and endothelial dysfunction, Hypertens., 38, 1400-1405 (2001)

8. P.G. Storozhuk, Enzymes of direct and indirect antiradical protection of red blood cells and their role in initiation of hemoglobin oxygenation, antibacterial protection and cell division processes, Intensive care bull., 3.8, 13 (2003)

9. T.A. Pushkina, E.S. Tokayev, T.S. Popova, E.N. Borodina, Superoxide-dismutase as a part of antioxidant therapy: the state of the issue and prospects, J. named after A.A. Lomonosov, N.V. Sklifosovsky "Emergency Medical Aid", 4, 42-47 (2016)

10. S.S. Kupchinskaya, Biological and pathogenetic role of antioxidant system in functioning of living organism, Togliatti Med. Consult., 1-2, 56-593 (2014)

11. T.V. Serota, M.V. Zakharchenko, M.N. Kondrashova, Cytoplasmic superoxide dismutase activity - a sensitive indicator of the state of antioxidant system of rat liver and brain, Biomed. Chem., 60(1), 63-71 (2014)
12. Y. Yamazaki, T. Takao, Metalation states versus enzyme activities of $\mathrm{Cu}, \mathrm{Zn}$-superoxide dismutase probed by electrospray ionization mass spectrometry, Anal. Chem., 80(21), 8246-8252 (2008)

13. V.E. Volykhina, E.V. Shafranovskaya, Superoxyddismutases: structure and properties (in Russian), Vestnik VGMU, 8(4), 1-18 (2009)

14. M. Majewska, M. Podsiad, H. Czeczot, B. GrytnerZięcina, Hymenolepis diminuta: Experimental studies on the antioxidant system with short and long term infection periods in the rats, Experim. Parasitol., 129(2), 158-163 (2011)

15. J.S. Seo, J.Y. Park, J. Choi, et al., NADPH Oxidase Mediates Depressive Behavior Induced by Chronic Stress in Mice, The J. of Neurosci., 32(28), 9690-9699 (2012)

16. M.B. Iversen, R.H. Gottfredsen, U.G. Larsen, et al., Extracellular superoxide dismutase is present in secretory vesicles of human neutrophils and released upon stimulation, Free Radical Biol. and Med., 97, 478-488 (2016)

17. K. Morales, M.N. Olesen, E.T. Poulsen, et al., The effects of hypochlorous acid and neutrophil proteases on the structure and function of extracellular superoxide dismutase, Free Radical Biol. and Med., 81, 38-46 (2015)

18. J. Zhang, B. Wang, H. Wang, H. He, C. Yu, Disruption of the superoxide anions-mitophagy regulation axis mediates copper oxide nanoparticles-induced vascular endothelial cell death, Free Radical Biol. and Med., 129, 268-278 (2018)

19. U. Forstermann, W.S. Sessa, Nitric oxide syntheses: regulation and function, Europ. Heart J., 33(7), 829-837 (2012)

20. D.J. Bonda, X. Wang, G. Perry et al., Oxidative stress in Alzheimer disease: a possibility for prevention, Neuropharmacol., 59(4-5), 290-294 (2010)

21. E. Tokuda, T. Nomura, S. Ohara, et al., A copperdeficient form of mutant $\mathrm{Cu} / \mathrm{Zn}$-superoxide dismutase as an early pathological species in amyotrophic lateral sclerosis, Biochim. et Biophys. Acta (BBA) - Molec. Basis of Disease, 1864(6), Part A, 2119-2130 (2018)

22. H. Fujita, et al., Reduction of renal superoxide dismutase in progressive diabetic nephropathy, $\mathrm{J}$. Am. Soc. Nephrol., 20(6), 1303-1313 (2009)

23. M.A. Lutskiy, T.V. Kuksova, M.A. Smelianets, Y.P. Lushnikova, Endogenous system of antioxidant protection activity in the process of organism life activity, Success. of modern sci., 12-1, 20-23 (2014)

24. A.A. Boldyrev, Oxidative stress and brain, Soros Educat. J., 7(4), 21-28 (2001)

25. Ran Qitao et al., Transgenic mice overexpressing glutathione peroxidase 4 are protected against oxidative stress-induced apoptosis, J. Biol. Chem. 53, 55137-55146 (2004)

26. K.K. Shulgin, T.N. Popova, T.I. Rakhmanova, Obtaining and properties of the glutathione 
peroxidase (in Russian), Applied biochem. and microbial., 44(3), 276-280 (2008)

27. N.V. Bezruchko, G.K. Rubtsov, N.B. Ganyaeva, et al., Catalysis of biological media of the human body and its clinical and biochemical termination in the evaluation of endotoxemia, Vestnik TSPU, 7(122), 94-98 (2012)

28. T.N. Popova, T.I. Rakhmanova, S.S. Popov, Medical enzymology, a textbook (Voronezh State University, Voronezh, 2008)

29. S.V. Uglanova, M.V. Popov, S.V. Kurova, et al., Stabilization of antioxidant enzymes in complexes and conjugates with block copolymers: treatment prospects for central nervous system diseases, Moscow university bulletin, ser. 2. Chemistry, 51(3), 227-23 (2010)
30. N.P. Chesnokova, E.V. Ponukalina, M.N. Bizenkova, General characteristics of sources of free radicals and antioxidant systems formation, Successes of modern sci., 7, 37-41 (2006)

31. M.A. Korolyuk, L.I. Ivanova, I.T. Mayorova, Method for determination of catalase activity, Lab., 1, 16-19 (1988)

32. D.I. Maksimovich, E.O. Korik, Investigation of the antioxidant enzyme activity in rats with the experimental metabolic syndrome, INTERNAUKA, 12-1(16), 10-12 (2017)

33. Guidance on the experimental (preclinical) study of new pharmacological substances, under the general editorship of R.U. Khabrieva, 2-published, revised and added (Medicine, Moscow, 2005) 